University of Wollongong

Research Online

Australian Institute for Innovative Materials -

Papers

Australian Institute for Innovative Materials

$1-1-2005$

A new magnetoplasmon sound wave in a two-dimensional electron gas under electromagnetic radiation

C Zhang

University of Wollongong, czhang@uow.edu.au

S Hessami Pilehrood

University of Wollongong

Follow this and additional works at: https://ro.uow.edu.au/aiimpapers

Part of the Engineering Commons, and the Physical Sciences and Mathematics Commons

Research Online is the open access institutional repository for the University of Wollongong. For further information contact the UOW Library: research-pubs@uow.edu.au 


\title{
A new magnetoplasmon sound wave in a two-dimensional electron gas under electromagnetic radiation
}

\author{
Abstract \\ We report a new magnetoplasmon mode and a sound-like collective excitation in two-dimensional \\ electronic systems under a weak magnetic field and a low-frequency electromagnetic radiation. The \\ analysis is based on the exact time-dependent wave functions and the exact relation between response \\ function with and without radiation. The new mode discovered here can be used as a generating \\ mechanism of high-frequency radiation from a system whose characteristic energy is low.

\section{Keywords} \\ magnetoplasmon, sound, wave, two, dimensional, electron, gas, under, electromagnetic, radiation \\ Disciplines \\ Engineering | Physical Sciences and Mathematics

\section{Publication Details} \\ Zhang, C. \& Pilehrood, S. (2005). A new magnetoplasmon sound wave in a two-dimensional electron gas \\ under electromagnetic radiation. Europhysics Letters: a letters journal exploring the frontiers of physics, \\ 69 (4), 623-628.
}


A new magnetoplasmon sound wave in a two-dimensional electron gas under electromagnetic radiation

This content has been downloaded from IOPscience. Please scroll down to see the full text. 2005 Europhys. Lett. 69623

(http://iopscience.iop.org/0295-5075/69/4/623)

View the table of contents for this issue, or go to the journal homepage for more

Download details:

IP Address: 130.130.37.84

This content was downloaded on 17/12/2013 at 04:34

Please note that terms and conditions apply. 
Europhys. Lett., 69 (4), pp. 623-628 (2005)

DOI: $10.1209 / \mathrm{epl} / \mathrm{i} 2004-10371-6$

\title{
A new magnetoplasmon sound wave in a two-dimensional electron gas under electromagnetic radiation
}

\author{
C. Zhang and S. Hessami Pilehrood \\ School of Engineering Physics and Institute of Superconducting and Electronic Materials \\ University of Wollongong - New South Wales 2522, Australia
}

received 19 August 2004; accepted in final form 1 December 2004

published online 14 January 2005

PACS. 77.22.Ch - Permittivity (dielectric function).

PACS. 78.20.Bh - Theory, models, and numerical simulation.

PACS. 78.30.Fs - III-V and II-VI semiconductors.

\begin{abstract}
We report a new magnetoplasmon mode and a sound-like collective excitation in two-dimensional electronic systems under a weak magnetic field and a low-frequency electromagnetic radiation. The analysis is based on the exact time-dependent wave functions and the exact relation between response function with and without radiation. The new mode discovered here can be used as a generating mechanism of high-frequency radiation from a system whose characteristic energy is low.
\end{abstract}

Recent experimental observation of zero-resistance state (ZRS) and magnetoresistance oscillation (MRO) in high-mobility two-dimensional electronic systems [1-3] has stimulated renewed interest in the transport properties of electronic systems under electromagnetic (EM) radiation [4-7]. Both MRO and ZRS are the consequence of the interplay of the photon frequency and the cyclotron frequency. Due to the resonant scattering of electrons by impurities under EM radiation, electrons can travel through the different orbits without loss of energy.

In this letter, we report an interesting property of the collective excitation in an electronic system under a weak magnetic field and a low-frequency EM radiation. It is well known that in a superfluid, the low-energy collective excitation exhibits a roton mode at small values of wave vectors $(q)$ and is a sound wave at large $q[8]$. This roton mode was later found in another ordered system, a two-dimensional electron gas at very low temperature and under very high magnetic field [9]. This was also known as the magneto-roton in a fractional quantum Hall state (FQHS). In what follows we shall show that for a normal two-dimensional electronic system under a weak magnetic field and a low-frequency EM radiation, a roton-like plasmon mode as well as a sound wave can be found when the radiation frequency is equal to the cyclotron frequency. Our theoretical prediction is based on a simple physical picture of resonant electron-EM field coupling. When this coupling is included exactly in the electronic states, one can set up an exact relation between the response function of the system under zero and finite radiation. This relation allows us to study various properties of the system and derive this plasmon mode.

(C) EDP Sciences 
Let us consider a two-dimensional electron gas in the $x-y$ plane. We choose the direction of the quantizing magnetic field to be the $z$-direction, $\boldsymbol{B}=B \boldsymbol{e}_{z}$, the radiation field to be along the $y$-direction, $\boldsymbol{E}(t)=E \sin (\omega t) \boldsymbol{e}_{y}$, where $E$ and $\omega$ are the amplitude and frequency of the radiation field. In the presence of a static magnetic field and a laser field, the Schrödinger equation for a single electron is given as

$$
i \hbar \frac{\partial}{\partial t} \psi(\boldsymbol{r}, t)=\frac{\left(\boldsymbol{p}-e \boldsymbol{A}_{1}-e \boldsymbol{A}_{\gamma}\right)^{2}}{2 m^{*}} \psi(\boldsymbol{r}, t)
$$

Here $m^{*}$ is the effective mass of the electron, $\boldsymbol{A}_{1}$ and $\boldsymbol{A}_{\gamma}$ are, respectively, the vector potentials for the static magnetic field and the radiation field. Equation (1) can be exactly solved [10], and the time-dependent wave function is written in terms of unitary transformations,

$$
\Psi(\boldsymbol{r}, t)=e^{i u_{1}(t)} e^{i x u_{2}} e^{i p_{x} u_{3}} e^{i p_{y} u_{5}} \exp \left[-i E_{n} t\right] \exp \left[i k_{y} y\right] \phi_{n}\left(x-x_{0}\right) .
$$

The unitary transformations can be derived analytically, with the initial condition $u_{i}(t=$ $0)=0$. The solutions are $[10]$

$$
\begin{aligned}
u_{1}(t)= & \frac{e^{2} E^{2}}{2 m^{*}}\left(\frac{1}{\omega_{c}^{2}-\omega^{2}}\right)^{2}\left[\frac{\omega_{c}^{3}}{2 \omega^{2}} \sin \left(2 \omega_{c} t\right)+\frac{\omega}{4}\left(\frac{3 \omega_{c}^{2}}{\omega^{2}}-1\right) \sin (2 \omega t)+\right. \\
& \left.+\left(\frac{\omega_{c}^{2}-\omega^{2}}{2}\right) t-\frac{\omega_{c}^{3}}{\omega^{2}}\left\{\sin \left[\left(\omega_{c}-\omega\right) t\right]+\sin \left[\left(\omega_{c}+\omega\right) t\right]\right\}\right], \\
u_{2}(t)= & e E\left(\frac{\omega_{c}}{\omega_{c}^{2}-\omega^{2}}\right)\left[\frac{-\omega_{c}}{\omega} \sin \left(\omega_{c} t\right)+\sin (\omega t)\right], \\
u_{3}(t)= & \frac{e E}{m^{*}} \frac{\omega_{c}}{\omega}\left(\frac{1}{\omega_{c}^{2}-\omega^{2}}\right)\left[-\cos \left(\omega_{c} t\right)+\cos (\omega t)\right], \\
u_{5}(t)= & \frac{e E}{m^{*}}\left(\frac{1}{\omega_{c}^{2}-\omega^{2}}\right)\left[\frac{\omega_{c}}{\omega} \sin \left(\omega_{c} t\right)-\sin (\omega t)\right] .
\end{aligned}
$$

At weak magnetic field and under the resonance, $\omega=\omega_{c}=e B / m^{*}$, we have, $u_{2}(t)=$ $e E \omega_{c} t, u_{3}(t)=\frac{e E}{2 m^{*}} t^{2}$, and $u_{5}(t)=\frac{e E}{\omega_{c} m^{*}} t$.

It is a difficult task to calculate the response function for a system whose electronic states are given in eq. (2). However, by employing a canonical transformation of the second quantized field operators for two-dimensional interacting electrons under a uniform static magnetic field and a laser field, an exact formula that transforms the density response function of the electrons under a radiation field, $\hat{D}_{\alpha \beta}^{A}\left(\boldsymbol{r}, \boldsymbol{r}^{\prime \prime} ; t, t^{\prime \prime}\right)$, to that of the electrons without the laser field, $\hat{D}_{\alpha \beta}^{B}\left(\boldsymbol{r}, \boldsymbol{r}^{\prime \prime} ; t, t^{\prime \prime}\right)$, is derived [11]:

$$
\hat{D}_{\alpha \beta}^{A}\left(\boldsymbol{r}, \boldsymbol{r}^{\prime \prime} ; t, t^{\prime \prime}\right)=\hat{D}_{\alpha \beta}^{B}\left(\boldsymbol{r}+\boldsymbol{u}(t), \boldsymbol{r}^{\prime \prime}+\boldsymbol{u}\left(t^{\prime \prime}\right) ; t, t^{\prime \prime}\right),
$$

where $\boldsymbol{u}(\boldsymbol{t})=u_{3}(t) \boldsymbol{e}_{x}+u_{5}(t) \boldsymbol{e}_{y}$. We assume that in the absence of the radiation field our system can be satisfactorily described by the random-phase approximation under a weak magnetic field. The response function for such a system can be readily obtained and is well documented:

$$
\begin{aligned}
\hat{D}_{\alpha \beta}^{B}\left(\boldsymbol{q} ; t, t^{\prime}\right)= & 1-V(q) 2 i \sum_{n k_{y}} \sum_{n^{\prime}} N_{n n^{\prime}}\left(q_{x}, q_{y}\right) N_{n n^{\prime}}\left(q_{x}, q_{y}\right) \times \\
& \times\left[f_{n, k_{y}}-f_{n^{\prime}, k_{y}+q_{y}}\right] e^{-i\left(\epsilon_{n}-\epsilon_{n^{\prime}}\right)\left(t-t^{\prime}\right)},
\end{aligned}
$$


where $f_{n, k_{y}}=\left[\exp \left[\epsilon_{n}-\mu\right]+1\right]^{-1}$ is the Fermi-Dirac distribution function, and $V_{q}=2 \pi e^{2} / q$ is the Coulomb interaction. $N_{n n^{\prime}}$ are form factors given as

$$
\begin{aligned}
\exp & {\left[i q_{x} l^{2} k_{y}\right] N_{n n^{\prime}}\left(q_{x}, q_{y}\right)=M_{n n^{\prime}}\left(q_{x}, k_{y}, k_{y}+q_{y} ; t\right) } \\
& =\int \mathrm{d} x \phi_{n k_{y}}\left(x-x_{0}+u_{3}(t)\right) e^{i q_{x} x} \phi_{n^{\prime} k_{y}^{\prime}}\left(x-x_{0}^{\prime}+u_{3}(t)\right)
\end{aligned}
$$

and $x_{0}^{\prime}=\left(k_{y}+q_{y}\right) l^{2}$. Making use of the properties of the harmonic oscillators, the above integration can be evaluated:

$$
\begin{gathered}
M_{n n^{\prime}}\left(q_{x}, k_{y}, k_{y}+q_{y}\right)=\sqrt{\frac{n_{<}}{n_{>}}} i^{n_{>}-n_{<}} \exp \left[i q_{x} l^{2}\left(k_{y}+q_{y} / 2\right)-i q_{x} 2 u_{3}(t)\right] \times \\
\quad \times \exp [-Y / 2] Y^{\left(n_{>}-n_{<}\right) / 2} L_{n_{<}}^{n_{>}-n_{<}}(Y)=\exp \left[i q_{x} l^{2} k_{y}\right] N_{n n^{\prime}}\left(q_{x}, q_{y}\right),
\end{gathered}
$$

where $Y=(1 / 2)(q l)^{2}$. We now perform the following operations: i) a spatial Fourier transformation to map the response to the real space; ii) shifting the coordinates according to eqs. (3)-(6) to obtain the response function $D^{A}\left(t, t^{\prime}\right)$ or $D^{A}(\tau, T)$, where $\tau=t-t^{\prime}$ and $T=t+t^{\prime}$; iii) carrying out $T$-average over one period of the radiation. The response function under the radiation can now be written as

$$
D^{A}(q, \Omega)=1+2 V(q) \sum_{n n^{\prime}}\left[N_{n n^{\prime}}\left(q_{x}, q_{y}\right)\right]^{2} \frac{\left|f_{n}-f_{n^{\prime}}\right|}{\omega_{x}} \ln \left[\frac{\omega_{p}^{2}-\left(m \omega_{c}-\omega_{x}+\omega_{y}\right)^{2}}{\omega_{p}^{2}-\left(m \omega_{c}+\omega_{x}+\omega_{y}\right)^{2}}\right],
$$

where $\omega_{n n^{\prime}}=\left|n-n^{\prime}\right| \omega_{c}, \omega_{x}=\pi q_{x} E / 2 B$ and $\omega_{y}=q_{y} E / B$.

Here the poles of $D^{A}(\boldsymbol{q} ; \Omega)$ are the modes of magnetoplasmons, $\omega_{p}$, given as

$$
1=\frac{2 r_{s}}{q \omega_{x}} \sum_{n m}\left[N_{n, n+m}\left(q_{x}, q_{y}\right)\right]^{2}\left|f_{n}-f_{n+m}\right| \ln \left[\frac{\omega_{p}^{2}-\left(m \omega_{c}-\omega_{x}+\omega_{y}\right)^{2}}{\omega_{p}^{2}-\left(m \omega_{c}+\omega_{x}+\omega_{y}\right)^{2}}\right] .
$$

In the above equation all energies are scaled by the zero-field Fermi energy $\epsilon_{F}$, all wave numbers are scaled by $k_{F}, r_{s}=m^{*} e^{2} / \kappa \hbar^{2} k_{F}$, and is the plasma parameter, where $\kappa$ is the dielectric constant of the host semiconductor. The $m=1$ term is the principal plasmon mode, and high $m$ terms are the Bernstein modes. Assuming the principal mode is well separated from the higher modes, we can obtain the following analytical solution for $\omega_{p}$ :

$$
\omega_{p}=\left[\frac{\left(\omega_{c}+\omega_{x}+\omega_{y}\right)^{2} \exp \left[q \omega_{x} / 2 r_{s} c_{1}\right]-\left(\omega_{c}-\omega_{x}+\omega_{y}\right)^{2}}{\exp \left[q \omega_{x} / 2 r_{s} c_{1}\right]-1}\right]^{1 / 2},
$$

where $c_{m}=\sum_{n}\left[N_{n, n+m}\left(q_{x}, q_{y}\right)\right]^{2}\left|f_{n, k}-f_{n+m, k+q}\right|$. It can be shown easily that in the limit of $E \rightarrow 0, \omega_{p}=\left(\omega_{q}^{2}(B)+\omega_{c}^{2}\right)^{1 / 2}$, where $\omega_{q}^{2}(B)=2 r_{s} c_{1} / q$, whose long-wavelength limit is $2 \pi n e^{2} q / m^{*}$. The dispersion and $B$-dependence of $\omega_{p}$ for the principal mode are plotted in figs. 1 and 2. We have used parameters for the GaAs semiconductor system: $m^{*}=0.067 m_{e}$, $r_{s}=1.0, \epsilon_{F}=11 \mathrm{meV}$ and $q_{y}=q / \sqrt{2}$. We have also introduced a dimensionless field amplitude, $R(E)=e E k_{F} / m^{*} E_{F}^{2}$.

It can be seen that the electron-photon resonant coupling has qualitatively changed the property of the magnetoplasmon. In the absence of the radiation field, the behavior of $\omega_{p}(E=$ $0)$ is well known. Under an EM radiation whose frequency is the cyclotron frequency, the plasma frequency increases rapidly with the inverse magnetic field in the low magnetic-field regime. The resonant radiation field not only significantly enhances the plasmon energy, it 


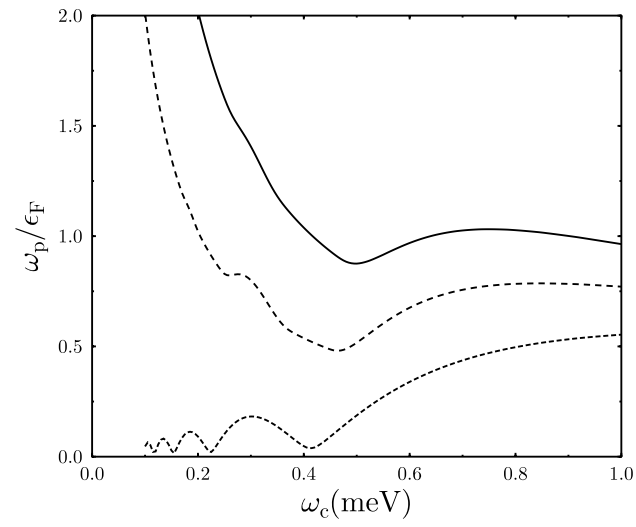

Fig. 1

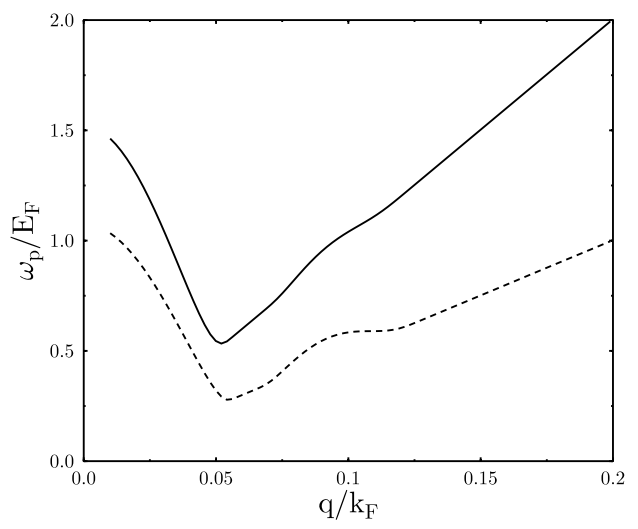

Fig. 2

Fig. 1 - Plasma energy as a function of the magnetic field for three different values of laser intensities, $R(E)=0.0$ (dotted line), $R(E)=0.1$ (broken line), and $R(E)=0.2$ (solid line). $q / k_{F}=0.1$. All other parameters are given in the text.

Fig. 2 - Plasma dispersion at fixed magnetic field and radiation frequency $\left(\omega=\omega_{c}=0.4 \mathrm{meV}\right)$, the radiation intensities are: $R(E)=0.1$ (broken line), and $R(E)=0.2$ (solid line).

also removes the magnetic oscillation in the low- $B$ regime. The dispersion of $\omega_{p}$ is given in fig. 2. The effect due to resonant coupling is most important in the finite- $q$ regime. The terms $\omega_{x}$ and $\omega_{y}$ are the dominant contributions in this regime. This leads to a minimum (roton-like) in $\omega_{p}$ in the low- $q$ regime and sound-like (linear in $q$ ) magnetoplasmon mode in large- $q$ regime. The speed of this sound-like wave is $E / B$ along the $y$-direction and slightly higher along the $x$-direction. At low $B$ and high $E$, this speed can be much higher than the Fermi velocity. For $E=10^{5} \mathrm{~V} / \mathrm{m}$ and $B=0.1 \mathrm{~T}$, the speed of the plasma wave is about 10 times $v_{F}$. This estimate is only valid when the static magnetic field is weak, but still much larger than the magnetic-field component of the radiation field. Therefore, the wave speed of the magnetoplasma sound derived here cannot be close to the value of $c$.

The magnetoplasma sound wave discussed here can be measured experimentally by farinfrared light scattering. The differential scattering cross-section is directly proportional to the dynamic structural function, which consists of two parts,

$$
S(\boldsymbol{q}, \omega)=\frac{\hbar}{2 \pi e^{2}} \epsilon_{I}(q, \omega) /|\epsilon(q, \omega)|^{2}+\frac{\hbar}{2 \pi e^{2}} \pi \delta\left(\epsilon_{R}(q, \omega)\right) .
$$

In the absence of exchange and correlation, and disorder-induced finite-lifetime effect, the spectral weight of the scattering cross-section is only located at collective excitation energies. For a specifically chosen wave vector, one can observe the predicted plasmon mode. Figure 3 shows the real and imaginary parts of the dielectric function and the structural function. It can be seen that the high-energy magnetoplasmon mode calculated here indeed carries the most spectral weight. It should also be pointed out that the cyclotron resonance $(\mathrm{CR})$ is very strong due to the condition that the cyclotron frequency matches exactly the microwave frequency. The very reason that a high-energy plasmon can exist is the strong and nonlinear electron-microwave coupling (or CR). Therefore, even the light scattering is performed at the plasmon energy which is much higher than the cyclotron energy; both the position and the oscillator strength of the scattering cross-section reflect the nature of strong electronmicrowave resonance. This situation is similar to that of MW-induced ZRS and MRO. 


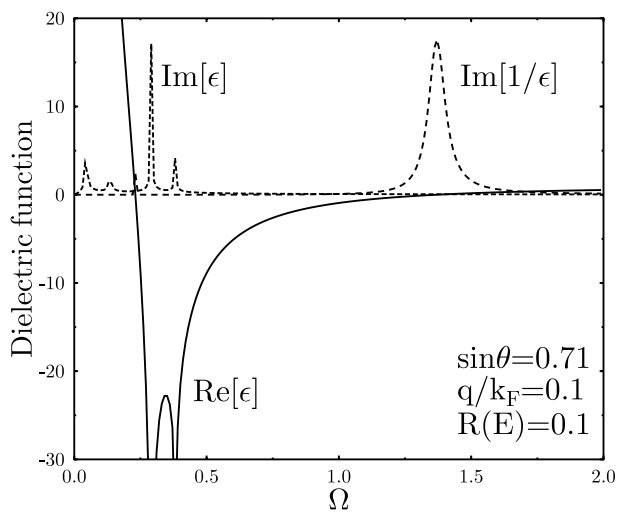

Fig. 3 - Frequency-dependent dielectric function for $\hbar \omega_{c}=1 \mathrm{meV}$, and $R(E)=0.1$.

Furthermore, because the collective excitation energy can be much higher than the characteristic energy of the system and of the radiation field, one can apply a resonant radiation field to the system to excite the high-energy plasmons. These high-energy plasmons can then be used to generate a high-frequency EM field, e.g., terahertz radiation. It should be pointed out that, as radiation-induced ZRS is entirely different to superconductivity, the roton-like structure and sound wave derived here are also entirely different to those in a FQHS. The former is due to resonant electron-field coupling and the latter is due to the system's finite ordering.

Finally, the electron-photon coupling also introduces a new intra-level plasma mode,

$$
\omega_{p}^{(0)}=\left[\frac{\left(\omega_{x}+\omega_{y}\right)^{2} \exp \left[q \omega_{x} / 2 r_{s} c_{0}\right]-\left(\omega_{x}-\omega_{y}\right)^{2}}{\exp \left[q \omega_{x} / 2 r_{s} c_{0}\right]-1}\right]^{1 / 2} .
$$

For both $q \rightarrow 0$ and $B \rightarrow 0$, the intra-level mode is given as

$$
\omega_{p}^{(0)} \approx\left[\frac{4 r_{s} c_{0} \omega_{x} \omega_{y}}{q}\right]^{1 / 2}
$$

For many-electron systems, the exchange-correlation effect becomes important when the characteristic interaction energy is greater than the characteristic kinetic energy. The characteristic interaction energy is $e^{2} \sqrt{n} / \kappa$, where $n$ is the $2 \mathrm{D}$ electron density and $\kappa$ is the dielectric constant of the host semiconductor. The characteristic kinetic energy is the Fermi energy. Now the ratio is given as $r_{s} / \sqrt{1.57}$, where $r_{s}$ is the plasma parameter defined in the text. For $r_{s}=1$, the ratio is less than 1. Actually, RPA is valid even for $r_{s}$ around 2 to 3 . This is the reason why we neglect the exchange-correlation effect. This situation will change if the magnetic field is strong. However, this is not the case here, since we are in the weak magnetic-field limit of $<1 \mathrm{~T}$. The dispersion curve of fig. 2 is for a magnetic field of around $0.25 \mathrm{~T}$.

In conclusion, we have shown that when a radiation field whose frequency equals the cyclotron frequency couples to an electronic system, the collective excitation of the system exhibits a magneto-roton mode at small $q$ and becomes a sound wave at large $q$. The new mode should be detectable in an optical experiment. 
This work is supported in part by the Australian Research Council (Discovery Scheme) and the University of Wollongong (Small Grant Scheme).

\section{REFERENCES}

[1] Mani R. G., Smet J. H., von Klitzing K., Narayanamuri V., Johnson W. B. and UmanSKY V., Nature, 420 (2002) 646.

[2] Zudov M. A., Du R. R., Pfeiffer L. N. and West K. W., Phys. Rev. Lett., 90 (2003) 046807.

[3] Ye P. D., Engel W., Tsui D. C., Simmons J. A., Wendt J. R., Vawter G. A. and Reno J. L., Appl. Phys. Lett., 79 (2001) 2193.

[4] Lei X. L. and Liu S. Y., Phys. Rev. Lett., 91 (2003) 226805.

[5] Durst Adam C., Sachdev Subir, Read N. and Girvin S. M., Phys. Rev. Lett., 91 (2003) 086803.

[6] Shi Junren and XIe X. C., Phys. Rev. Lett., 91 (2003) 086801.

[7] Ryzhit V. and Vyurkov V., Phys. Rev. B, 68 (2003) 165406.

[8] Henshaw D. G. and Woods A. D. B., Phys. Rev., 121 (1961) 1266.

[9] Girvin S. M., MacDonald A. H. and Platzman P. M., Phys. Rev. Lett., 54 (1985) 581.

[10] Fujita M., Toyoda T., Cao J. C. and Zhang C., Phys. Rev. B, 67 (2003) 075105.

[11] Toyoda T., Fujita Maho, Koizumi H. and Zhang C., Phys. Rev. B, 71, 15 January (2005). 